J3eA, Journal sur l'enseignement des sciences et technologies de l'information et des systèmes, Volume 2, Hors-Série 2, 3 (2003)

DOI : http://dx.doi.org/10.1051/bib-j3ea:2003603

(C) EDP Sciences, 2003

Machines moléculaires : mécanique des molécules

G. Rapenne

Groupe NanoSciences, CEMES-CNRS

29 rue Jeanne Marvig

F-31055 Toulouse Cedex 4, France

rappennedcemes. fr 


\title{
Machines moléculaires : Mécanique des molécules
}

\author{
Dr Gwénaël RAPENNE \\ Groupe NanoSciences, CEMES-CNRS \\ 29 rue Jeanne Marvig, F-31055 Toulouse Cedex 4
}

E-mail:.rapennedcemes.fr

\section{Machines et moteurs moléculaires}

Dans l'objectif constant de miniaturisation, la conception de machines et de moteurs à l'échelle moléculaire est en pleine expansion. Les chimistes, inspirés par les machines moléculaires naturelles que sont par exemple les enzymes, élaborent des composants ayant des fonctionnalités de plus en plus complexes et variées. Dans cet exposé sera tout d'abord présentée l'ATP synthase, formidable machine biologique dans laquelle se produit un mouvement contrôlé de rotation puis, s'en inspirant, nous verrons quels nano-dispositifs les chimistes ont pu imaginer.

\section{Introduction}

Une machine moléculaire est un système multicomposant dans lequel un signal provoque le mouvement d'un des composants. Ce signal peut être électronique, lumineux, contrôlé par le pH. Ce mouvement doit être réversible, d'une amplitude suffisamment large pour qu'il soit visible et utilisable. On distingue 2 familles de machines, les machines qui travaillent de manière linéaire (ex. muscle) ou rotative. Cet exposé se restreindra aux machines où tout ou partie de la molécule subit un mouvement de rotation.

\section{Machines moléculaires rotatives}

Dans le domaine des nanosciences, un des challenges tant conceptuel que synthétique est la conception et la construction d'un moteur moléculaire de taille nanométrique [1]. Un moteur est une machine qui de manière continue transforme une énergie en produisant un travail via un mouvement de rotation unidirectionnel contrôlé. L'exemple le plus connu est l'ATP Synthase [2].

\subsection{L'ATP synthase : un moteur rotatif biologique}

Chaque homme consomme en moyenne $40 \mathrm{~kg}$ d'ATP par jour. L'ATP est converti en ADP en libérant un phosphate et de l'énergie puis le stock d'ATP est reconstitué par le mécanisme inverse. Le siège de ces réactions est une enzyme: l'ATP synthase. Elle transforme en énergie chimique l'énergie électrique due à une différence de concentration en protons (ions $\mathrm{H}^{+}$) de part et d'autre d'une membrane, par le biais de la synthèse d'ATP.

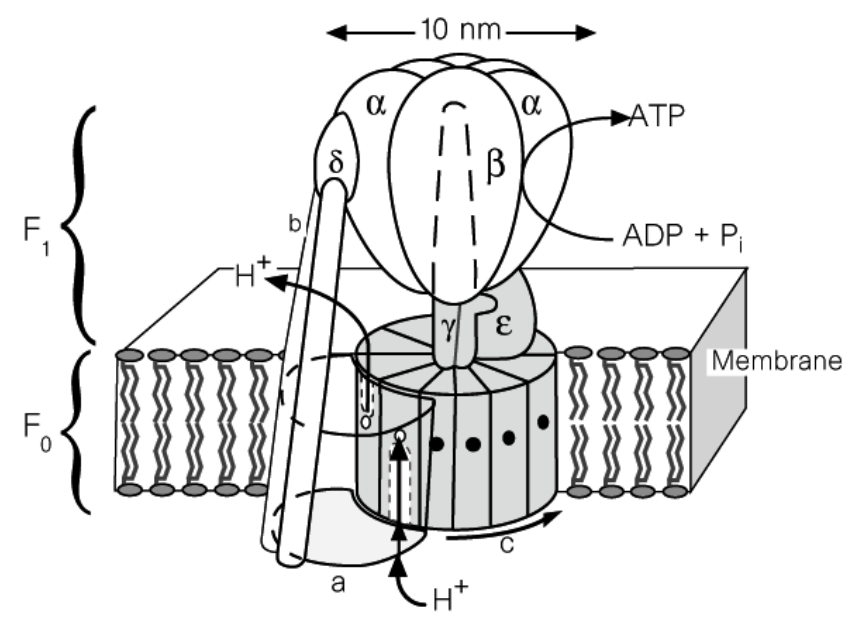

Figure 1. Schéma de l'ATP synthase incorporée dans une membrane.

Comme le montre la figure ci-dessus, elle est constituée de deux parties : la première, nommée $\mathrm{F}_{0}$, est incorporée dans la membrane des cellules ; la seconde, nommée $\mathrm{F}_{1}$, est à l'extérieur. $\mathrm{F}_{0}$ est composée d'une partie mobile, le rotor, (c) constitué de 10 à 12 sousunités protéiques, et d'un stator (b). Près de ce dernier, quand un proton se fixe sur une des sous-unités c chargées négativement, celle-ci devient neutre et se déplace pour rejoindre un environnement avec lequel elle a plus d'affinité, celui de la membrane, vers qui elle se tourne en entraînant l'ensemble. Le rotor, une sorte de turbine à protons se prolonge dans $F_{1}$ par un arbre ( $\square$ ), les mouvements de cette partie au sein de $\mathrm{F}_{1}$ activent tour à tour les trois sous-unités qui synthétisent l'ATP.

Ce qui est extraordinaire c'est que ce mécanisme ne tourne que dans un sens pour produire de l'ATP et il est réversible, la turbine tournant dans l'autre sens afin de produire de l'énergie en consommant l'ATP présent lorsque le besoin s'en fait sentir.

Inspirés par les performances de cette molécule naturelle, nous allons maintenant voir les premières machines moléculaires artificielles synthétisées. 


\subsection{L'hexa-tert-butyl décacyclène : un rotor monomoléculaire}

Le premier rotor vraiment moléculaire à avoir été étudié est la molécule A ci-dessous [3]. Il s'agit de l'hexatert-butyl décacyclène, une plateforme aromatique de 1.5 $\mathrm{nm}$ de diamètre reposant sur six pieds tert-butyl, chaine carbonée saturée permettant d'isoler la plateforme aromatique de la surface métallique sur laquelle elle a été étudiée.
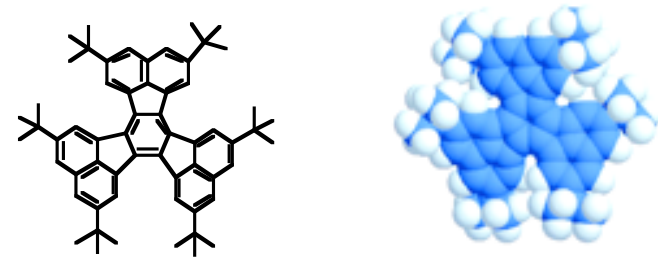

Figure 2. La molécule A

Lorsqu'on utilise un microscope à effet tunnel, on a la possibilité de visualiser les molécules, mais aussi de les manipuler. Sur la figure 3, on voit une monocouche de cette molécule sur une surface de cuivre $(\mathrm{Cu} 100)$.

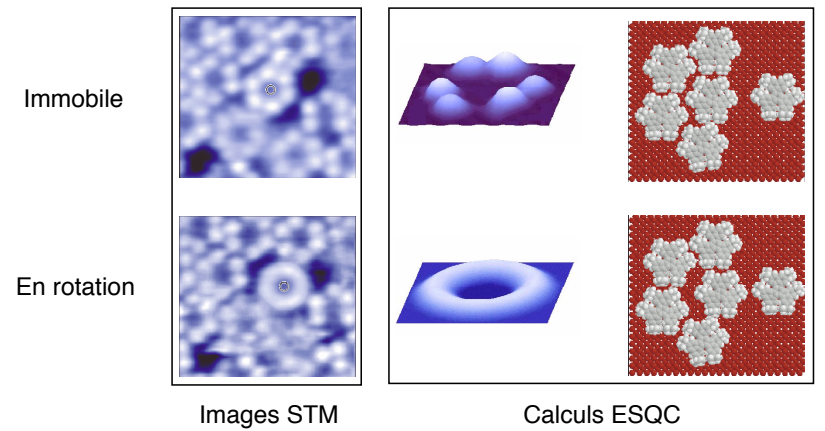

Figure 3. Monocouches de molécules A ; Images STM et images calculées représentant une molécule immobile (en haut) et en rotation (en bas).

La molécule à laquelle on s'intéresse est au centre. On voit que lorsqu'on la déplace légèrement vers la droite, la molécule, initialement immobile (taches blanches bien localisées) se met à tourner. Les différentes parties visibles de la molécule ne sont plus localisées et on ne voit plus qu'un tore. On a donc bien un rotor monomoléculaire, mais sa rotation est contrôlée par son environnement. Sans ce réseau supramoléculaire, la molécule perd sa fonction, de plus, cette rotation n'est pas directionnelle.

\section{3 : Synthèse d'un moteur moléculaire transformant un courant électrique en mouvement de rotation unidirectionnel}

L'étape suivante consiste à avoir un rotor qui joue ce rôle indépendamment de son environnement. Des moteurs moléculaires ont déjà été proposés et même synthétisés, mais leur comportement n'a jamais étudié à l'échelle moléculaire. Le moteur que l'on va présenter ici a été pensé pour être déposé sur une surface entre deux électrodes distantes de quelques dizaines de nanomètres.

Ce moteur est composé de trois parties. La première est un socle constitué de trois pieds (type tabouret de piano), fonctionnalisé de manière à se lier de manière covalente à la surface isolante dans laquelle seront enterrées les deux électrodes de la nanojonction. La seconde est une plateforme aromatique ayant 5 groupements électroactifs accrochés de manière linéaire et rigide. Entre ces deux parties, un métal de transition jouant le rôle de rotule. La partie supérieure sera libre de tourner alors que la partie inférieure restera immobile, accrochée à la surface. Le schéma de principe est représenté ci-dessous.

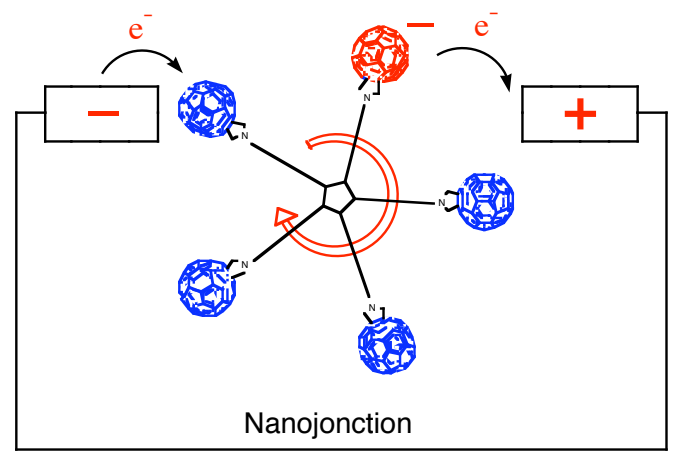

Figure 4. Une molécule entre les deux électrodes constituant la nanojonction

Les groupements électroactifs sont des fragments [60]fullerène. Celui à proximité de la cathode devrait être réduit et, comme l'a montré $\mathrm{L}$. Kouwenhoven pour une molécule de $\mathrm{C}_{60}$ entre deux électrodes [4], repoussé, conduisant à la rotation d'un cinquième de tour de la partie supérieure de la molécule. Le fragment réduit (chargé négativement, en rouge) devrait se rapprocher de l'anode et y céder son électron. Entre temps, un autre groupement électroactif devrait alors se placer au voisinage de la cathode, il serait alors réduit et ainsi de suite.

Nous devrions donc observer la conversion d'un flux d'électrons en mouvement de rotation. Ce mouvement devrait même être unidirectionnel si la molécule est placée de manière dissymétrique entre les deux électrodes.

\section{Machines combinant les mouvements de translation et de rotation :}

Translation [5] ou rotation [3] d'une molécule ont déjà été effectuées dans notre équipe. Ce que nous allons présenter ici, c'est le design et la synthèse des premières molécules combinant un mouvement de translation et de rotation. C'est le cas des brouettes moléculaires [3]. 


\section{1: Design et synthèse de brouettes moléculaires}

Une molécule analogue à la brouette macroscopique que nous connaissons est représentée ci dessous [6].
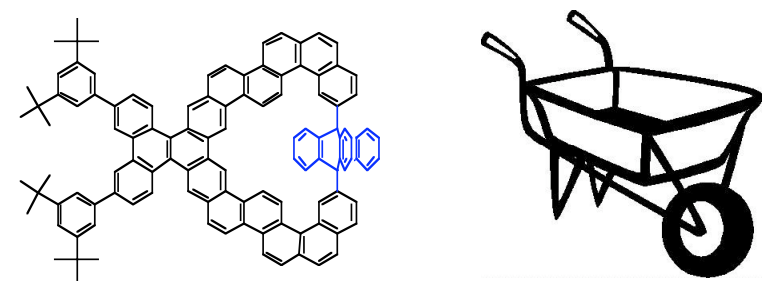

Figure 5. Une brouette moléculaire et son modèle macroscopique

La molécule contient une roue triptycène (en bleu) encastrée dans un châssis polyaromatique. Cette molécule était un point de départ, mais elle n'a pas été synthétisée. On touche en effet ici un point délicat du design de nouvelles molécules, leur faisabilité. Dans ce cas, elle n'est pas synthétisable, ou en tout cas pas dans des délais convenables vis-à-vis de nos collaborateurs physiciens. Après un travail préliminaire autour de la méthodologie de synthèse, deux molécules ont été proposées et synthétisées, elles sont représentées figure 6.
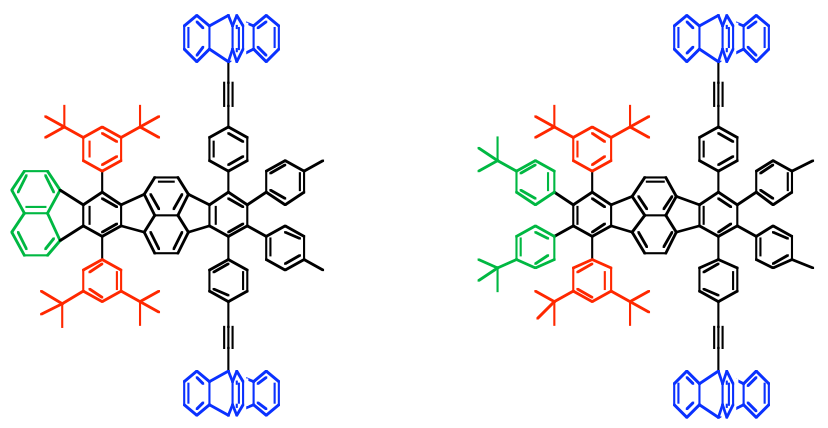

Figure 6.Les deux brouettes moléculaires synthétisées

Celle de gauche a été obtenue après 14 étapes de synthèse avec un rendement global de $2 \%$, celle de droite après 15 étapes et avec un rendement global de $1.3 \%$ (soit une moyenne par étape de 75\%) [7].

On peut voir en bleu les roues (fragments triptycènes, composés de 3 pales à $120^{\circ}$ l'une de l'autre), en rouge les deux pieds et en vert la partie où on va agir avec la pointe $\mathrm{du}$ microscope à effet tunnel pour manipuler la nanobrouette (la poignée en quelque sorte).

\section{2: Modélisation du comportement de brouettes moléculaires}

Des calculs théoriques ont montré d'une part que les roues triptycènes pouvaient tourner d'autre part que le choix de la surface était très délicat, sa rugosité jouant un rôle primordial. La figure 7 montre la rotation d'un tiers de tour sous l'action de la pointe du microscope. Une des trois pales du triptycène est représentée en bleu, et la molécule est vue de profil.

Il est intéressant de noter qu'un design basé sur des concepts et des réflexes macroscopiques conduit au résultat attendu au niveau nanométrique, ce n'est, très souvent, pas le cas.

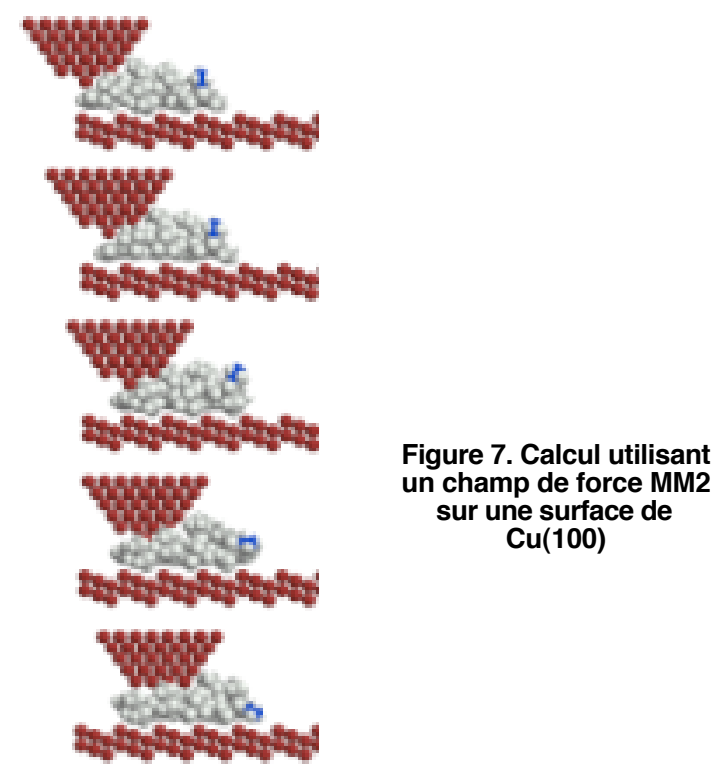

\section{Perspectives}

Ces molécules vont être étudiées sur des surfaces métalliques avec un microscope à effet tunnel pour les brouettes moléculaires, et sur des surfaces isolantes avec un microscope à force atomique pour le moteur à cinq pales.

\section{Remerciements}

Je tiens à remercier Jean-Pierre Launay et Alexandre Carella pour leur participation à la conception et à la synthèse du moteur rotatif ainsi que Christian Joachim, Hao tang et Gorka Jimenez-Bueno pour leur travail sur le sujet Nanobrouettes.

\section{Références}

[1] (a) Special Issue on Molecular Machines, Acc. Chem. Res. 2001, 34, 409-522.

(b) J-P. Launay, L'Act. Chim., 2001, 6, 33-38.

[2] J. F. Walker, Angew. Chem. Int. Ed. 1998, 37, 23082319.

[3] J. K. Gimzewski, C. Joachim, R. Schlitter, V. Langlais, H. Tang, Science 1998, 281, 531-533.

[4] L. Kouwenhoven, Nature 2000, 407, 35-36.

[5] F. Moresco, G. Meyer, K.H. Rieder, H Tang, A. Gourdon, C. Joachim, Phys. Rev. Lett 2001, 86, 672-677.

[6] C. Joachim, H. Tang, F. Moresco, G. Rapenne, G. Meyer, Nanotechnology 2002, 13, 330-335.

[7] G. Jimenez-Bueno, G. Rapenne, Chem. Commun. 2003 , soumis pour publication. 